\title{
LIDERAZGO EDUCATIVO Y RETOS DE LA EDUCACIÓN SUPERIOR EN EL SIGLO XXI: HACIA LA VISIÓN Y LA ACCIÓN
}

En el presente artículo se desarrollan algunas ideas y propuestas para el ejercicio de un liderazgo educativo que pueda asumir los principales retos de la educación superior en el siglo XXI. Se retoman en esta reflexión los compromisos de las universidades en la construcción de sociedades más justas y equitativas acordes a las necesidades y exigencias de un momento histórico caracterizado por los escenarios de la globalización y el desarrollo científico tecnológico. Se destacan por lo tanto, los vínculos permanentes entre la educación y el desarrollo social y económico; se retoman los planteamientos formulados en la Conferencia Mundial sobre la Educación Superior en 1998, los cuales evidencian los alcances e implicaciones de los principales desafios que enfrenta la educación en el siglo XXI, por lo cual es de vital importancia un tipo de liderazgo que responda efectivamente a estos retos mediante acciones beligerantes, oportunas, participativas y concertadas
In this article, we are developing some ideas and proposals for an educational leadership exercise in order to assume higher education's main challenges in the XXI centurn: In this reflection we are taking into consideration the universities' commitments in the construction of more fair and equitable societies according to its necessities and demands in a historical moment distinguished by globalization and scientific technological development. Therefore, we have to emphasize the permanent links between education and economic and social development; besides, we take back some of the ideas presented at the higher education world conference in 1998. which evidenced the implications of the main challenges that education faces in the XXI century. For that reason it is of vital importance, a type of leadership that responds effectively. to those challenges through belligerent, participative and precise actions.

* Licenciada en Sociología de la Facultad de Ciencias Sociales de la Universidad Nacional. Post Grado en Educación en el Centro de Investigación y Docencia (CIDE). Académica de la Universidad Nacional en la Sede Regional Chorotega y en la Maestría en Educación con Mención en la Enseñanza del Inglés. Ha desempeñado los cargos de Subdirectora, Coordinadora del Programa de Estudios Generales y como investigadora en la Sede Regional Chorotega de la Universidad Nacional, actualmente coordina la Maestría en Educación con mención en la enseñanza del Inglés en la Sede Regional Chorotega. 


\section{Presentación}

Este espacio tiene como objetivo fundamental abordar el tema del liderazgo educativo, no desde la abstracción misma del término, sino introduciéndolo y visualizando sus potencialidades en la gestión universitaria; la cual ha estado inmersa en las últimas décadas, en contextos caracterizados por una situación de crisis y ante un escenario de retos y desafíos a los cuales es inminente dirigirse mediante una acción concertada, oportuna y pertinente, que posibilite hacer efectiva la trascendencia de la universidad en los procesos de desarrollo nacional, local y regional.

Por esto, cabe preguntarse ¿qué tan pertinente y necesario es el liderazgo en la gestión universitaria? ¿qué clase de liderazgo se requiere?, ¿Es necesario un liderazgo democrático y participativo? ¿ Cómo enfrentar coyunturas históricas, que requieren cambios y transformaciones institucionales de frente a las demandas y necesidades de la sociedad civil y política?.

A partir de este marco se pretende en el desarrollo del presente artículo, ir configurando una red de relaciones que permitan hacer una lectura, sobre la relevancia y la concepción misma de liderazgo que podría efectivamente coadyuvar en la configuración y construcción de soluciones y estrategias que posibiliten atender por un lado, los diversos y complejos problemas que han aquejado a las instituciones de educación superior, y por otro lado, atender los muchos desafíos educativos que se enfrentan en la actualidad.

A continuación se tratará, en un primer momento, de contextualizar los referentes y los escenarios donde cobra sentido y significado la acción de un liderazgo educativo y, en un segundo momento, se retomará este tema de frente a los retos institucionales que deben atenderse en el siglo XXI.

No se pretende por supuesto, dar por agotado el tema, sino por el contrario provocar el análisis y la reflexión sobre la relación gestión universitaria y liderazgo educativo, en particular en los contextos latinoamericanos, y con especial atención al caso costarricense.

\section{Universidad y sociedad: Un compromiso para el desarrollo}

\section{Educación y dinámica social}

La educación es un fenómeno básicamente social, de manera que es resultante del momento histórico en que acontece, del lugar donde se realice, de la cultura imperante y de la estructura sociopolítica vigente. 
En el transcurso de la historia. se han elaborado multiplicidad de definiciones que expresan el sentido. finalidad y naturaleza de la educación; lo cual evidencia lo complejo del término Sarranova (1997, p.27) aborda precisamente este punto al indicar las diversas connotaciones asignadas al término educación. por ejemplo al asociársele a una institución social, es decir, al sistema educativo institucionalizado, también concibiéndosela como producto o resultado de una acción, por lo cual se habla de "buena" o "mala" educación, de una educación adaptada o no a las exigencias de los tiempos, de una educación conservadora o progresista,etc. Además, se ha concebido como proceso que relaciona de manera prevista o imprevista a dos o más seres humanos, situándose en una relación de intercambio y de influencias recíprocas.

Por consiguiente, el precisar el concepto en el pleno de su significación. exhaustividad e implicaciones, deja manifiesto, que la educación como parte del desarrollo social asume de forma explícita todas las connotaciones que caracterizan las ideas imperantes en el campo intelectual, social, político y cultural de las sociedades, según el espacio y tiempo en el que se les ubiquen

Si consideramos los fines de la educación en este marco, estos pueden responder a ciertas funciones generales, por ejemplo, la referencial, puesto que la eficacia del proceso educativo depende de los fines que se persigan, la organizadora atiende a la forma en que se realizan las acciones educativas, la integradora, según sea como los fines se establezcan y como responden a un eje aglutinador de todo el proceso educativo, y finalmente la prospetiva, anticipando el resultado previsible, con lo cual se determinan reglas y acciones. (Sarramona 1997 p 37).

De manera que, los fines de la educación no son fortuitos, sino que responden a factores sociales característicos de determinadas épocas, culturas, coyunturas o situaciones. Por esto la educación es un sistema que forma parte de un sistema mayor que es precisamente la sociedad, bajo el principio dinámico de la intervención para el cambio y la transformación.

El marco anterior, permite entonces afirmar el sentido de construcción social que tiene inherentemente el ser de la Educación, es decir, su significación histórica y su construcción cotidiana cobran sentido desde esta perspectiva colectiva, dinámica, y dialéctica.

\section{Educación superior y desarrollo}

Partiendo de la anterior posición, la educación superior se constituye en las sociedades modernas en una de las más importantes fuerzas para incorporar la sociedad al desarrollo integral, y si se quiere al desarrollo sostenible. La educación en general y especialmente la superior se convierte en una importante 
inversión que debe protegerse y desarrollarse, en tanto promueve y es generadora de la potencialidad humana que se requiere para la transformación y el desarrollo de la sociedad.

Así, la Universidad como inversión debe analizarse en éstas dimensiones, que incluyen lo sociopolítico y lo sociocultural, en la dimensión de los aspectos sociopolíticos dado que todas las estrategias de desarrollo reconocen el papel del hombre y sus potencialidades como fundamental actor y dinamizador de las mismas; y en lo sociocultural es evidente hoy mas que nunca, que el conocimiento es el elemento central para el desarrollo sostenible de las sociedades, y por lo tanto quedar al margen de él significa sentenciarse a la marginalidad, a la exclusión del crecimiento económico, no-sólo en lo referido a los aspectos productivos, sino que compete a cualquier expresión de la actividad y vivencia humana.

Es decir, la educación superior se ha convertido en la necesidad misma para enfrentar el futuro, sin lo cual la sociedad actual no podría superar las formas tradicionales de integración del individuo a los procesos de producción, comunicación e información interpuesto por el fenómeno mismo de la globalización, entendida ésta más allá de la dimensión económica, sino que de una u otra manera ésta ha permeado la cultura, la sociedad, las formas de convivencia y de comunicación, entre otros aspectos de la vida humana.

De manera entonces que, a grandes rasgos, puede decirse que la función esencial de la educación es el desarrollo continuo de la persona y las sociedades, no como la fórmula milagrosa, sino como una vía, al servicio de un desarrollo humano más armonioso, más genuino, para hacer retroceder la pobreza, la exclusión, , la intolerancia, las opresiones, las guerras, etc. Por lo tanto la utopía deseada, es poder alcanzar el nivel necesario de desarrollo económico y social sostenible y racional desde el punto de vista del medio ambiente, una creatividad cultural nutrida por un conocimiento y una comprensión mejor del patrimonio cultural y social, un nivel de vida digno fundado en los derechos humanos, la democracia, la tolerancia y el respeto mutuo.

\section{Los sistemas de educación superior y los nuevos escenarios educativos actuales: posibilidades para el ejercicio de un liderazgo educativo proactivo}

\section{Enfrentando retos}

La educación superior enfrenta en muchos países de América Latina desafíos y dificultades relativos entre otros a la financiación, a las condiciones de acceso a los estudios, a capacitación del personal, la formación basada en las 
competencias, la mejora y conservación de la calidad de la enseñanza, la investigación y los servicios, la pertinencia o actualización de los planes de estudio, las posibilidades reales de empleo con los diplomas otorgados, el establecimiento de acuerdos de cooperación eficientes y la igualdad de acceso a los beneficios que reporta la cooperación internacional, por citar algunos. Castro, I. (1997) . Torres, R. ( 1999).

Así mismo, se debe hacer frente a los retos que suponen las nuevas oportunidades que abren las tecnologías que mejoran las maneras de producir, organizar, difundir y controlar el saber y el acceder al mismo. Haddad, W. (1997) pero a su vez es relevante garantizar un acceso equitativo a estas tecnologías en todos los niveles de los sistemas de enseñanza. Si bien es cierto, en la segunda mitad del siglo XX, la educación superior se expande de forma espectacular, es cierto también, que con ella se ha agudizado aún más la disparidad, la inequidad que ya era enorme, entre los países industrialmente desarrollados y en particular los países menos desarrollados, lo cual en lo que respecta al acceso a la educación superior y la investigación y los recursos de que se disponen. Tunnermann,C. (1998). De igual forma, las últimas décadas han estado caracterizadas por un aumento de las diferencias de oportunidades de enseñanza en muchos de nuestros países latinoamericanos, con las repercusiones que esto tiene, ya que al carecerse de educación y de investigación, se imposibilita el desarrollo potencial crítico y creativo de las personas y no se podrá garantizar un auténtico desarrollo endógeno y sostenible; no pudiéndose acortar la distancia que nos separa de los países industrializados.

De manera que, el intercambio de conocimientos, la cooperación internacional, y las nuevas tecnologías pueden constituirse en oportunidades que posibiliten reducir estas disparidades.

Pero al mismo tiempo, se requieren de acciones concretas por parte de las instituciones de educación superior, que permitan un replanteamiento de su ser y hacer de frente a las demandas y necesidades sociales, donde éstos nuevos escenarios requieren la participación de una universidad proactiva, formadora de mentes críticas e innovadoras y capaces de visualizar y participar en la construcción del desarrollo con un amplio sentido de solidaridad y equidad.

Así, durante los últimos años se ha insistido de sobremanera en los procesos de evaluación institucional que le permita a las universidades, repensar y replantear su gestión. En el caso particular, por ejemplo de la Universidad Nacional de Costa Rica, estas tareas se han emprendido desde mediados de la década del noventa, con miras a realizar esfuerzos institucionales dirigidos a evaluar los Currículos y demás actividades dirigidas a la investigación, la extensión y la producción. 
En este sentido, se han realizado importantes esfuerzos tendientes a replantear el quehacer académico con miras a responder a los cambios del entorno social, económico, político. y tecnológico. Cabe anotar, sin embargo. que estas acciones emprendidas en el ámbito institucional deben tener como norte también, entre otros aspectos. la transformación de las estructuras organizativas que posibilite disponer de una plataforma de apoyo ágil, oportuna y pertinente a las necesidades académicas sobre las cuales se asienta el desarrollo institucional.

En otras palabras, es una tarea altamente prioritaria disponer de formas y estructuras organizacionales que faciliten el cumplimiento efectivo de la misión y funciones de la educación superior. Lo anterior cobra especial atención al tomarse en cuenta los planteamientos formulados en la Declaración Mundial de la Educación Superior en octubre de 1998 en París, donde se señalan los grandes desafíos que han de enfrentarse en el presente siglo. UNESCO (1998, pág. 4-)

A continuación se precisan algunos:

1) Formar profesionales altamente calificados y ciudadanos responsables, capaces de atender a las necesidades de todos los aspectos de la actividad humana. ofreciéndoles cualificaciones que estén a la altura de los tiempos modernos, comprendida la capacitación profesional, en las que se combinen los conocimientos teóricos y prácticos de alto nivel mediante cursos y programas que estén constantemente adaptados a las necesidades presentes y futuras de la sociedad

2) Construir un espacio abierto para la formación superior que propicie el aprendizaje permanente, brindando una óptima gama de opciones y la posibilidad de entrar y salir fácilmente del sistema, así como oportunidad de realización individual y movilidad social con el fin de formar ciudadanos que participen activamente en la sociedad y estén abiertos al mundo, y para promover el fortalecimiento de las capacidades endógenas y la consolidación de un marco de justicia de los derechos humanos, el desarrollo sostenible, la democracia y la paz.

3) Promover, generar y difundir conocimientos por medio de la investigación y, como parte de los servicios que ha de prestar a la comunidad, promocionar las competencias técnicas adecuadas para contribuir al desarrollo cultural, social y económico de las sociedades fomentando y desarrollando la investigación científica y tecnológica al lado de la investigación en el campo de las ciencias sociales, las humanidades y las artes creativas.

4) Contribuir a comprender, interpretar, preservar, reforzar, fomentar y difundir las culturas nacionales y regionales internacionales e históricas, en un contexto de pluralismo y diversidad cultural.

5) Contribuir a proteger y consolidar los valores de la sociedad, velando por inculcar en los jóvenes los valores en que reposa la ciudadanía democrática 
y proporcionando perspectivas críticas y objetivas a fin de propiciar el debate sobre las opciones estratégicas y el fortalecimiento de enfoques humanistas.

6) Contribuir al desarrollo y la mejora de la educación en todos los niveles, en particular mediante la capacitación del personal docente.

Además de lo anterior se consideró por todos los miembros participantes a esta conferencia mundial sobre educación superior, UNESCO (1998. pág.5-20) la urgencia de forjar una visión caracterizada por los siguientes grandes lineamientos:

a. Igualdad al acceso a los estudios superiores

b. Fortalecimiento de la participación y promoción del acceso a las mujeres

c. Promoción del saber mediante la investigación en los ámbitos de la ciencia. el arte y las humanidades y la difusión de sus resultados

d. Pertinencia de la educación superior, es decir, mejor articulación con los problemas de la sociedad y del mundo del trabajo, reforzar sus funciones de servicio a la sociedad

e. Reforzar la cooperación con el mundo del trabajo y el análisis y la previsión de las necesidades de la sociedad

f. La diversificación como medio de reforzar la igualdad de oportunidades

g. Desarrollar métodos educativos innovadores, que propicien un pensamiento crítico y creativo.

h. Garantizar la excelencia de la enseñanza y la investigación.

En este gran marco se señala también la relevancia significativa que tiene el asumir como gran reto el potencial y los desafíos de la tecnología. Esto por cuanto los rápidos progresos de estas nuevas tecnologías de la información y la comunicación, han estado modificando la forma de elaboración, adquisición y transmisión de conocimientos, además, brindan la posibilidad de renovar el contenido de los cursos y los métodos pedagógicos, y de ampliar el acceso a la educación superior.

Por esto, se acuerda en esta conferencia mundial establecer como una tarea indispensable el aprovechamiento de las ventajas y el potencial de las nuevas tecnologías de la información y la comunicación en los procesos de formación educativa.

A partir de todo lo anterior se puede visualizar algunos de los muchos retos y desafíos que deben enfrentar los sistemas de educación superior en nuestros países.

Las tareas dirigidas a superar los problemas orgánicos y estructurales que obstaculizan esta visión de futuro han iniciado en muchas instituciones con procesos tendientes a reformas institucionales pero: ¿Cómo propiciar en las comunidades universitarias una actitud y una clara conciencia de la importancia de participar en estos procesos de forma responsable y comprometida? ¿ Cómo se 
podrán hacer concretar y hacer efectivas las acciones correctivas que permitan alcanzar esta visión de futuro hacia donde debe dirigirse la educación superior?

¿ Cómo lograr construir un proyecto educativo universitario que esté a la altura de las necesidades y demandas de nuestro tiempo?.

Estas interrogantes forman parte también de la lista de desafíos que deben enfrentarse en este nuevo siglo.

\section{Hacia un contenido del liderazgo educativo}

El concepto de liderazgo ha través del tiempo ha adquirido una serie de connotaciones mediadas y matizadas por cosmovisiones de mundo, propias de la cultura y la comunicación entre otras formas de intercambio y construcción de significados.

De manera que el término está cargado de valores y entendido desde diferentes prismas, convirtiéndose en un concepto multivalente pero que tienden a un referente común. Algunos ejemplos de esto se evidencian en frases como las siguientes: "Este niño es todo un líder, porque sus compañeritos hacen lo que él les dice”, “... Se necesitan personas agresivas, que sepan dirigir proyectos de impacto, dueñas de sí mismas, verdaderos líderes." "Los líderes de ahora ya no son los de antes ya no saben lo que quieren ni saben hacia donde van", " el maestro debe ser un líder con autoridad moral para tomar decisiones y hacerlas respetar" "... No hay un liderazgo, no hay una cabeza pensante, por eso todo anda muy mal...". Estas frases evidencian que el estereotipo más clásico es identificar al líder, con la figura de un caudillo o con aquella persona extraordinariamente dotada de cualidades y habilidades que es capaz de dirigir multitudes, pronunciar discursos cargados de emotividad y esperanza que tiendan a la consecución de un fin. Da la sensación de que el liderazgo es un "don" que sólo algunas personas poseen; lo cual invisibiliza la riqueza de nuestra naturaleza humana como seres creativos dotados de toda una subjetividad que orientada a fines nobles y de empresa social, pueden rendir extraordinarios resultados.

Pero ¿Cómo entender el concepto de liderazgo y concretamente de liderazgo educativo en medio del escenario que se ha descrito anteriormente? Un líder y en especial un líder educativo, a juicio personal, es aquel que se incorpora con toda su entrega, capacidad y potencialidad humana, a la búsqueda y construcción de proyectos sociales que tiendan a lograr sociedades más justas, equitativas y solidarias. Es aquel que con su actitud positiva logra trascender las palabras a los hechos modelando conductas y acciones. No basta con los discursos y los deseos. 
En este sentido la obra de Roger Fisher y Alan Sharp, (1999) "El Liderazgo Lateral", nos enfrenta precisamente a la visión de un liderazgo cuya viabilidad existe en tanto, se consideren una serie de condiciones, que propicien y promuevan acciones tendientes, a mejorar sustancialmente cualitativa y cuantitativamente la calidad en las relaciones interpersonales, entre los miembros que conforman una determinada organización, a fin en última instancia de garantizar el éxito y la consecución de los fines que se persiguen. Es decir, orienta en cuanto desarrollar ciertas capacidades y destrezas donde haya un reconocimiento personal del potencial individual que todos tenemos, pero que se requiere a la vez desarrollar la forma de agudizar la habilidad de pensar, de razonar mejor, de saber comunicarnos, de poder configurar estrategias para buscar organizadamente resultados, entre otros aspectos destacados por lo autores. En síntesis se requieren ciertas condiciones que permitan ejercer un liderazgo lateral. Pero todo esto, tiene como centro garantizar la calidad en las relaciones de intercambio y comunicación social, en mejorar la capacidad de construir no autocráticamente sino bajo el principio de la cooperación, lo cual se revertirá indudablemente en el éxito o no de una empresa u organización. Es decir, se transciende la visión caudillezca y totalitaria de desempeño en el mundo social del trabajo y la convivencia humana en general.

Por lo tanto, entonces personalmente, se parte de la idea de que el liderazgo es una actitud y virtud humana mediante la cual se puede alcanzar y lograr determinados fines, pero que requiere de ciertas condiciones, que deben gestarse y propiciarse para poder ejercerlo a favor de una meta o interés común.

\section{Liderazgo educativo y gestión universitaria}

En los apartados anteriores se realizó un bosquejo breve y general sobre la situación actual que enfrenta la educación superior, evidenciándose la necesidad de un liderazgo que posibilite concretar en la práctica los cambios requeridos que le permitan a las universidades ejercer el cometido social sobre el cual se legitima y sustenta su razón de ser. Este liderazgo con visión de construcción conjunta y solidaria hacia un fin común, cobra vital importancia en momentos en los cuales se debe trascender del discurso a la práctica, construyendo alternativas y estrategias, participando y asumiendo con responsabilidad y compromiso las tareas que demandan estos nuevos retos de la educación superior. Pero la tarea empieza con cada uno de los funcionarios que laboran tanto desde la docencia y la investigación y la extensión, como aquellos que ocupan cargos eminentemente administrativos. Es decir, en este contexto de crisis y retos de la educación superior hablar de liderazgo lateral es pertinente, pero a la vez nos 
enfrenta a otro desafío, se deben propiciar y promover los espacios institucionales que permitan reactivar o generar actitudes para el cambio. No basta con el discurso hay que ejercitarse en la acción, retomar la importancia de participar positiva y constructivamente en la configuración de soluciones.

En momentos como los actuales la participación y el liderazgo educativo en el ámbito universitario, son vitales; pero se debe abonar en la consecución de un liderazgo académico a la altura de las exigencias que se tienen en la actualidad. Por lo cual se cree en la conveniencia de generar proyectos institucionales tendientes a esta formación, en los futuros profesionales, pero también en los funcionarios universitarios. Muchas veces damos por sentado que el liderazgo es una cualidad heredada genéticamente, o adquirida por un deseo y una actitud. éstas son fundamentales pero no suficientes. Este tipo de proyecto puede concretarse en el caso de los funcionarios universitarios de diversas formas, mediante foros, conferencias, u otras actividades promovidas al interior de las diversas unidades académicas. En la formación de futuros profesionales mediante los diferentes planes de estudio, ya sean como cursos específicos o como ejes curriculares. En el caso concreto de los funcionarios universitarios, es importante a propósito de los procesos de reforma, disponer de líderes que coadyuven a la tarea de construir un proyecto universitario, el cual sea identificado como una construcción colectiva, y no producto de la toma de decisión de unos cuantos a quienes se les ha otorgado o cedido el derecho absoluto de decidir por todos los demás.

En este sentido, se visualiza un líder educativo, con una visión crítica y con habilidad y conocimientos necesarios para crear y facilitar espacios para la participación y el cambio. Es decir, tal y como lo afirma Grinberg (1999) modelar acciones que evidencien el entendimiento, el conocimiento, la visión, los hábitos de pensamiento y acción. También la disposición de indagar, cuestionar y problematizar, la inclinación a tomar riesgos y a experimentar y evaluar consecuencias, las habilidades para crear espacios y prácticas que sean cuidadosas, dedicadas, respetables y respetuosas, confiables, estimulantes, preocupadas, y que contribuyan a desarrollar comunidades de aprendizaje donde se avance en la importancia de la democracia, la equidad, la diversidad y la justicia social.

Es la identificación de un liderazgo capaz de articular, conceptualizar, crear y promover espacios y posibilidades para un cambio crítico y efectivo de las condiciones que inhiben el mejoramiento de todos (as) y para todos (as). De ahí que un líder educativo debe potencialmente ser un visionario, que evalúa y analiza las consecuencias de sus acciones, que aprende a escuchar, a dialogar, a investigar a buscar evidencias antes de tomar decisiones o emitir juicios de valor 
de forma precipitada. que demanda condiciones que aseguren la participación amplia y constante. pero con respeto y posibilidades de crear de construir conjuntamente; aprovechando las diferencias para enriquecer la visión de conjunto. Este liderazgo educativo es el que se ejercita y se gesta en un aprender a conocer, a ser y a vivir, para revertirlo en un aprender a hacer. En tiempos como los actuales es imperativo entender la importancia de la organización, de la planificación institucional, entender los sistemas y juegos de poder, los significados de los cambios y las implicaciones de ciertas adaptabilidades sociales e institucionales.

En fin un líder educativo hoy más que nunca no puede permanecer neutral y distante, la construcción del proyecto universitario es responsabilidad de todos y en esta medida los esfuerzos para potencializar ese liderazgo lateral, desde las posiciones estudiantiles, la docencia, la investigación, la extensión. así como desde la administración, es una tarea altamente prioritaria.

\section{Anotaciones Finales}

Al iniciar el siglo XXI, la Educación Superior está enfrentando retos sin precedentes bajo el impacto de la globalización, el crecimiento económico basado en el conocimiento, y la revolución de la comunicación y la información. Estos retos pueden ser percibidos como amenazas o grandes oportunidades y posibilidades, las instituciones educativas congruentes con la finalidad de aportar al desarrollo y a la construcción de un proyecto de sociedad más equitativa, justa y solidaria, deben estar dispuestas a tomar ventaja de estas oportunidades, de promover y desarrollar acciones proactivas en el lanzamiento de reformas e innovaciones significativas.

La tarea está orientada a formular una visión y a desarrollar una gestión que posibilite que las instituciones de educación superior cumplan su cometido y asuman la responsabilidad y el compromiso social de contribuir efectivamente al desarrollo de cada país. En este sentido hablar de liderazgo educativo adquiere una trascendencia histórica por cuanto, estos retos y desafíos sólo se pueden asumir, mediante la participación activa y el ejercicio positivo y beligerante de un liderazgo que potencialice las diferencias y logre construir, mediante la negociación, el consenso, el análisis cuidadoso de las posibilidades, de las alternativas y estrategias; en síntesis sistemas de educación superior capaces de hacer efectiva su misión de contribuir al desarrollo nacional en su sentido mas amplio y profundo. 


\section{Referencias}

Aguilar, M. La Misión del al Universidad Latinoamericana ayer y hoy: Revista Ciencias Sociales. XLII (83) 23-35.

Alfaro, G. (Compilador) Universidad Nacional. Maestría en Educación (20001) Compendio de Lecturas. Curso de Liderazgo Académico. Heredia, Costa Rica.

Banco Interamericano de Desarrollo (1994). A la bisqueda del siglo XXI: Nuevos caminos de desarrollo en Costa Rica.

Castro I. (1997) El pragmatismo Neoliberal y las desigualdades educativas en América Latina. Revista Mexicana de Sociología. 49 (3) 189-205.

Colegio de Licenciados y Profesores en Letras, Filosofía, Ciencias y Artes (1996). Desafios de la educación costarricense en la formación del ser Humano para la sociedad del Nuevo Siglo. En Memoria II Congreso Nacional de Educación.

Diario Reflexivo Curso Liderazgo. (2001) Notas Personales: Construyendo un Concepto Heredia, C.R.

Fisher, R. Sharp Alan. (1999). El Liderazgo Lateral. Bogotá, Colombia: Grupo Editorial Norma.

Grinberg J. (1999). Desafios y Posibilidades para el Futuro de la Educación. El Papel del Docente Líder. Eduforum @utdt.eduConferencia

Hadad,W.D. La Globalización de la Economía: Repercusiones en la Educación y en la Formación de Competencias. Revista Perspectivas, Vol. XXVII N.1, marzol997.

Sarramona J. (1997). Fundamentos de Educación. Madrid, España; Editorial CEAC S.A.

Torres, R. (1999).Nuevo Rol docente: Que modelo de formación, para que modelo educativo. Revista Paraguaya de Sociología. Año36 N. 105 Mayo-Agosto 1999, p.7-28

Tunnermann ,C (1998). Transformación de la Educación Superior: Retos y Perspectivas. Heredia ,Costa Rica, Editorial Universidad Nacional. 
UNESCO. Conferencia Mundial sobre Educación Superior: La Educación Superior en el Siglo XXI. (1998) París, Francia.

Weiler, H (1996) ¿Porqué fracasan las reformas? La política educativa en Francia y en la República Federal de Alemania. En Revista Universidad Futura. 2 (4) 34-44. 